\title{
ON THE AUTOMATIC SOLUTION OF NONLINEAR FINITE ELEMENT EQUATIONS
}

\author{
Klaus-Jürgen Bathe and Eduardo N. Dvorkin \\ Department of Mechanical Engineering, Massachusetts Institute of Technology, Cambridge, MA 02139 ,
} U.S.A.

\begin{abstract}
An algorithm for the automatic incremental solution of nonlinear finite element equations in static analysis is presented. The procedure is designed to calculate the pre- and post-buckling/collapse response of general structures. Also, eigensolutions for calculating the linearized buckling response are discussed. The algorithms have been implemented and various experiences with the techniques are given.
\end{abstract}

\section{INTRODUCTION}

The nonlinear finite element analysis of structures requires the use of accurate and reliable finite element models and, of equal importance, the use of efficient procedures for the solution of the incremental equations of motion. The equation solution procedures are efficient when, for a given solution accuracy, the computer cost of solution is low and the solution is obtained in a reliable manner with a minimum amount of effort by the analyst.

Since the need for nonlinear analysis of structures has significantly increased during the recent years and will continue to do so, and since ever more complex problems are being tackled, much emphasis is currently being placed on the development of more general and automatic solution schemes.

The objective in this paper is to describe an algorithm that we have studied for a more automatic solution of the finite element nonlinear equations of motion. We assume in this paper that an appropriate finite element representation to idealize the physical problem has been selected and we are only concerned with the solution of the governing equations. Using the notation of Ref. [1], these equations are

$$
{ }^{i+\Delta} \mathbf{R}-{ }^{i+\Delta t} \mathbf{F}=\mathbf{0}
$$

where ${ }^{t+\Delta} \mathbf{R}$ is a vector of externally applied nodal point loads, and ${ }^{t+\Delta_{t}} \mathbf{F}$ is a vector of nodal point forces equivalent (in the virtual work sense) to the internal element stresses, both being evaluated at time $t+\Delta t$.

In this paper we consider only the static response of structures. Hence, if for the finite elements time independent constitutive relations are employed, the time variable is merely denoting a load level. However, the time steps have to be chosen judiciously when the material law is time-dependent, as in the analysis of creep problems.

The usual incremental solution of eqn (1) results in the following iterative scheme ([1], p. 490),

$$
{ }^{\tau} \mathbf{K}^{(t-1)} \Delta \mathbf{U}^{(t)}={ }^{t+\Delta} \mathbf{R}-{ }^{t+\Delta} \mathbf{F}^{(t-1)}
$$

where ${ }^{\tau} \mathbf{K}^{(t-1)}$ is a coefficient matrix and $\Delta \mathbf{U}^{(i)}$ is an increment to the current displacement vector,

$$
{ }^{t+\Delta t} \mathbf{U}^{(i)}={ }^{t+\Delta t} \mathbf{U}^{(t-1)}+\Delta \mathbf{U}^{(t)}
$$

The coefficient matrix ${ }^{\tau} \mathbf{K}^{(t-1)}$ is different in the various procedures used. In the full Newton-Raphson technique and the BFGS method, the matrix is updated in every iteration, whereas in a modified Newton iteration, the matrix is only updated at certain times. Line searches can also be effective using each of these methods, and it is clearly possible to combine Newton and quasi-Newton iterations and linc searches in one step-by-step solution scheme.

A study of the advantages and disadvantages of various solution procedures was reported in [2]. However, in that earlier work two major assumptions were made. First, we assumed that the analyst prescribes the various load levels for which the equilibrium configurations are to be calculated. This can be difficult without an approximate knowledge of the load carrying capacity of the structure. Second, we assumed that only the response up to the collapse of the structure was sought; i.e. the post-collapse response was not required. However, in some analyses the response after the (or a) critical point has been reached is of interest. By critical point we refer to a bifurcation point or limit (collapse) point.

In this paper we address the above two issues and describe an algorithm that we have implemented and studied to automatically choose appropriate load steps and calculate the pre- and post-collapse response of a structure. In the next section we present this algorithm which traces out the complete equilibrium path of the structure. For some classes of problems, to only estimate the collapse load, it can be effective to perform a linearized buckling analysis and in Section 3 we briefly discuss the solution of such problems. Some valuable experiences obtained with the solution techniques in the analyses of various problems are presented in Section 4, and in Section 5 we briefly conclude with some thoughts towards a further refinement of the automatic solution strategies.

\section{INCREMENTAL SOLUTION ALGORITHM}

In the analysis we assume that the structure is subjected to a proportionally varying load. In this case the basic equations to be solved are, based on eqn (2),

$$
{ }^{t} \mathbf{K} \Delta \mathbf{U}^{(i)}={ }^{t+\Delta t} \lambda \mathbf{R}-{ }^{t+\Delta} \mathbf{F}^{(t-1)}
$$


where $1+\Delta t \lambda$ is a load factor that describes the intensity of the reference load vector $\mathbf{R}$ to be applied at time $t+\Delta t$. The difficulty lies in defining the load factor for each load step $\Delta t, 2 \Delta t, \ldots$ prior to the incremental analysis. Without sufficient knowledge of the structural behavior, the analyst may prescribe too small increments in load, making the solution unduly expensive, or load increments that are too large, resulting in convergence difficulties during the equilibrium iterations. Also, the most effective solutionmeasured on the required computational effort-is obtained when variable load step sizes are used. Frequently, it is of significant advantage to employ large load steps initially and smaller load steps as the collapse load is approached. Then, after the peak load has been traversed, the load level must be decreased appropriately to solve for the post-collapse response.

Since our primary emphasis in the development of the algorithm was so far the automatic selection of the load increments, the algorithm uses eqn (4) in a modified Newton iteration only, in which the stiffness matrix is updated at any stage of the solution when the convergence is slow. However, it is realized that a full Newton iteration, a quasi-Newton iteration and line searches may increase the overall effectiveness of the solution technique.

\subsection{Load constraints}

The essence of the automatic algorithm that we have studied lies in the automatic selection of the incremental load levels, and the iteration with the load level and the displacements. The basic idea of iterating in the load-displacement space was researched earlier by a number of investigators, see e.g. Refs. [3-11].

When the iteration is performed in the loaddisplacement space, eqn (4) is used in the form

$$
{ }^{\tau} \mathbf{K} \Delta \mathbf{U}^{(t)}=\left({ }^{t+\Delta r} \lambda^{(i-1)}+\Delta \lambda^{(i)}\right) \mathbf{R}-{ }^{t+\Delta} \mathbf{F}^{(t-1)}
$$

and an additional equation is employed to constrain the length of the load step

$$
f\left(\Delta \lambda^{(t)}, \Delta \mathbf{U}^{(t)}\right)=0 .
$$

Several constraint equations of this form have been proposed such as the tangent constant arc-length [3] and the spherical constant arc-length techniques $[5,7]$.

In our research we found that for an automatic algorithm it is effective to use two different constraints depending on the response and load level considered; namely, the spherical constant arc-length and a constant increment of external work.

We use the spherical constant arc-length in the response regions far from the critical points, and in this case eqn (6) is

$$
\begin{gathered}
\left\{\left({ }^{(+\Delta t} \lambda^{(t-1)}-{ }^{(\lambda)}+\Delta \lambda^{(i)}\right\}^{2}+\mathbf{U}^{(t)} \mathbf{U}^{(t)}=\Delta l^{2}\right. \\
\mathbf{U}^{(t)}={ }^{t+\Delta t} \mathbf{U}^{(l)}-{ }^{t} \mathbf{U}
\end{gathered}
$$

where $\Delta l$ is the arc-length.

$\dagger\|\mathbf{a}\|$ denotes the Euclidian norm of the vector $\mathbf{a}[1]$.
The scheme of constant increment of external work is used near the critical points. In this case eqn (6) is for the first iteration,

$$
\left({ }^{(} \lambda+\frac{1}{2} \Delta \lambda^{(1)}\right) \mathbf{R}^{T} \Delta \mathbf{U}^{(1)}=W
$$

and for the next iterations,

$$
\left({ }^{(+\Delta t} \dot{\lambda}^{(t-1)}+\frac{1}{2} \Delta \lambda^{(1)}\right) \mathbf{R}^{T} \Delta \mathbf{U}^{(t)}=0
$$

where $W$ is the amount of external work in the step (and is positive or negative).

\subsection{Iterations within a load step}

Prior to the start of the incremental solution, the analyst specifies three items.

(1) The user inputs the reference load distribution, which corresponds to the vector $\mathbf{R}$ in eqn (5). This load distribution is varied proportionally during the analysis, but can be due to distributed and concentrated loads.

(2) The user specifies the displacement at a node corresponding to the first load level (i.e. corresponding to $\left.{ }^{\Delta} \lambda\right)$. We denote this displacement as ${ }^{\Delta} U_{k}^{*}$. Here it is deemed that to start the incremental solution it is easier to specify the displacement at a node, that the user selects, than the intensity $\left({ }^{\Delta t} \lambda\right)$ of the loads.

(3) The displacements corresponding to time $\Delta t$ determined by the specified displacement ${ }^{\Delta}{ } U_{k}^{*}$ also limit the size of any subsequent load change per step, because the user specifies a constant $\alpha$ and the algorithm assures that

$$
\|\mathbf{U}\| \leq \alpha\left\|^{\Delta r} \mathbf{U}\right\| \dagger
$$

where $\mathbf{U}$ is the displacement increment in any load step and ${ }^{\Delta t} \mathbf{U}$ are the displacements corresponding to time $\Delta t$.

2.2.1 Solution for the equilibrium configuration at time $\Delta t$ with prescribed displacement ${ }^{\Delta_{i}} U_{k}^{*}$. For this first load step, eqn (5) reduces to

$$
{ }^{0} \mathbf{K} \Delta \mathbf{U}^{(t)}=\left({ }^{\Delta t} \lambda^{(t-1)}+\Delta \lambda^{(t)}\right) \mathbf{R}-{ }^{\Delta t} \mathbf{F}^{(t-1)}
$$

with

$$
{ }^{\Delta} \lambda^{(0)}=0 ;{ }^{\Delta} \mathbf{F}^{(0)}=\mathbf{0} .
$$

The solution is obtained using the scheme discussed in [6]. For $i=1$, we use

$$
\begin{gathered}
{ }^{0} \mathbf{K} \Delta \mathbf{U}^{(1)}=\mathbf{R} \\
{ }^{\wedge} \lambda^{(1)}=\frac{{ }^{\Delta t} U_{k}^{*}}{\Delta U_{h}^{(1)}} ;{ }^{\wedge} \mathbf{U}^{(1)}={ }^{\Lambda}{ }^{\prime}{ }^{(1)} \Delta \mathbf{U}^{(1)} .
\end{gathered}
$$

Then for $i=2,3, \ldots$ we use two equations instead of eqn $(10)$,

$$
\begin{aligned}
& { }^{0} \mathbf{K} \Delta \overline{\mathbf{U}}^{(t)}={ }^{\Delta(} \lambda^{(t-1)} \mathbf{R}-{ }^{\Delta t} \mathbf{F}^{(r-1)} \\
& { }^{0} \mathbf{K} \Delta \overline{\mathbf{U}}^{(i)}=\Delta \lambda^{(i)} \mathbf{R}
\end{aligned}
$$

where we note that $\Delta \overline{\mathbf{U}}^{(i)}=\Delta \hat{\lambda}^{(i)} \Delta \mathbf{U}^{(1)}$. Since the displacement at degree of freedom $k$ is imposed we have 
the condition

$$
\Delta \hat{\lambda}^{(i)}=-\frac{\Delta \bar{U}_{k}^{(i)}}{\Delta U_{k}^{(1)}}
$$

and then

$$
\begin{gathered}
{ }^{\Delta} \lambda^{(i)}={ }^{\Delta} \lambda^{(1-1)}+\Delta \lambda^{(t)} \\
\Delta \mathbf{U}^{(t)}={ }^{\Delta} \mathbf{U}^{(1-1)}+\Delta \mathbf{U}^{(i)} \\
\Delta \mathbf{U}^{(t)}=\Delta \mathbf{U}^{(t)}+\Delta \lambda^{(t)} \Delta \mathbf{U}^{(1)} .
\end{gathered}
$$

Convergence of the iteration is reached when $[1,2]$

$$
\frac{\Delta \mathbf{U}^{(i)} r\left(\Delta t \lambda^{(1-1)} \mathbf{R}-{ }^{\Delta} \mathbf{F}^{(i-1)}\right)}{\Delta \mathbf{U}^{(1) \tau}\left({ }^{\Delta t} \lambda^{(1)} \mathbf{R}\right)} \leq \text { ETOL }
$$

where ETOL is an energy convergence tolerance.

2.2.2 Solutions for the equilibrium configurations at times $2 \Delta t, 3 \Delta t, 4 \Delta t, \ldots$, using the spherical constant arc-length algorithm. At the beginning of each such solution we calculate the load-step length $\Delta l$,

$$
\Delta l=\beta \sqrt{\mathbf{U}^{\tau} \mathbf{U}+\lambda^{2}}
$$

where

$$
\mathbf{U}={ }^{\prime} \mathbf{U}-{ }^{\imath-\Delta} \mathbf{U} ; \lambda={ }^{\prime} \lambda-{ }^{t}-\Delta t \lambda
$$

and the constant $\beta$ scales the previous load step length to an appropriate current load step length as discussed in eqns (30) and (31)

Using eqn (5) we now obtain for iteration $i=1$,

$$
\begin{aligned}
& { }^{t} \mathbf{K} \Delta \overline{\mathbf{U}}^{(1)}={ }^{\prime} \lambda \mathbf{R}-\mathbf{F} \\
& { }^{\mathrm{t}} \mathbf{K} \Delta \overline{\mathbf{U}}^{(1)}=\mathbf{R}
\end{aligned}
$$

and

$$
\begin{aligned}
\Delta \mathbf{U}^{(1)} & =\Delta \overline{\mathbf{U}}^{(1)}+\Delta \lambda^{(1)} \Delta \overline{\mathbf{U}}^{(1)} \\
{ }^{t+\Delta} \mathbf{U}^{(1)} & =\mathbf{U}+\Delta \mathbf{U}^{(1)} ;{ }^{t+\Delta} \lambda^{(1)}={ }^{\prime} \lambda+\Delta \lambda^{(1)}
\end{aligned}
$$

where the load increment $\Delta \lambda^{(1)}$ is determined from the current load step length, i.e. eqn (7),

$$
\Delta \mathbf{U}^{(1)^{r}} \Delta \mathbf{U}^{(1)}+\left(\Delta \lambda^{(1)}\right)^{2}=\Delta l^{2}
$$

For iterations $i=2,3, \ldots$ we use the solution of eqn (22), and in addition we solve

$$
{ }^{\top} \mathbf{K} \Delta \mathbf{U}^{(i)}={ }^{t+\Delta^{i}} \lambda^{(i-1)} \mathbf{R}-{ }^{t+\Delta t} \mathbf{F}^{(t-1)}
$$

so that

$$
{ }^{i+\Delta} \mathbf{U}^{(i)}={ }^{t+\Delta t} \mathbf{U}^{(t-1)}+\Delta \mathbf{U}^{(i)}+\Delta \lambda^{(i)} \Delta \overline{\mathbf{U}}^{(1)}
$$

Equation (7) now yields $\Delta \lambda^{(i)}$, and we then have

$$
{ }^{1+\Delta t} \lambda^{(i)}={ }^{t+\Delta t} \lambda^{(i-1)}+\Delta \lambda^{(i)}
$$
(19)

Convergence in the iteration is measured as in eqn

$$
\frac{\Delta \mathbf{U}^{(i) T}\left({ }^{t+\Delta t} \lambda^{(t-1)} \mathbf{R}-{ }^{t+\Delta t} \mathbf{F}^{(t-1)}\right)}{\Delta \mathbf{U}^{(1) T}\left(\Delta \lambda^{(1)} \mathbf{R}\right)} \leq \text { ETOL. }
$$

We note that $\Delta \lambda^{(i)}$ is obtained from a quadratic equation, so that different situations can arise.

(a) We do not obtain any real roots. In this case we restart from the last established equilibrium position using the constant increment of external work algorithm (see Section 2.2.3).

(b) We obtain two real roots. In this case we use the root for which $\gamma$ is largest[7], where

$$
\gamma=\mathbf{U}^{(t-1)^{\tau}} \mathbf{U}^{(t)}
$$

Once a new equilibrium configuration has been determined, we check whether the condition in eqn (9) is satisfied. If eqn (9) is not satisfied we restart from the previous equilibrium configuration using

$$
\left.\Delta l\right|_{\text {new }}=\left.\Delta l\right|_{\text {old }} \frac{\|\mathbf{U}\|_{\text {allowable }}}{\|\mathbf{U}\|_{\text {actual }}} .
$$

If eqn (9) is satisfied, we proceed with the solution for the next load increment using

$$
\left.\Delta l\right|_{\text {new }}=\left.\Delta l\right|_{\text {old }} \sqrt{\frac{N_{1}}{N_{2}}} \frac{\|\mathbf{U}\|_{\text {allowable }}}{\|\mathbf{U}\|_{\text {actual }}}
$$

where $N_{1}$ is the optimum number of iterations and $N_{2}$ is the number of iterations that were used in the previous load step increment.

We found the above scheme to be effective as long as the solution is not too close to a critical point, at which time it is more efficient to use the scheme of constant increment of external work. The measure used to decide whether to switch from the constant arc-length to the constant increment of external work scheme is the value of ${ }^{t} W /{ }^{-\Delta t} W$. When this value is close to unity, i.e. when $1-\delta \leq\left|{ }^{t} W /^{\prime-\Delta^{t}} W\right|<1+\delta$ where $\delta$ is small, the algorithm uses the constant increment of external work scheme.

2.2.3 Solutions for the equilibrium configurations at times $2 \Delta t, 3 \Delta t, 4 \Delta t, \ldots$, using the constant increment of external work algorithm. At the beginning of each step we calculate

$$
{ }^{t+{ }^{t}} W=\beta^{\prime}{ }^{\prime} W
$$

where ' $W$ corresponds to eqn ( $8 \mathrm{a}$ ) and $\beta$ ' is a constant which we set equal to $\lambda t^{t-\Delta} \lambda$.

The iteration is now performed as for the scheme using the spherical constant arc-length (see Section 2.2.2) but with eqns (8a) and (8b) to determine the increments $\Delta \lambda^{(i)}$. (8a).

For the first iteration we use eqns (21)-(23) and eqn

For iterations $i=2,3,4, \ldots$ we use eqns (22), (25) (27) and (8b), and convergence is measured by eqn (28).

In these iterations we always obtain two real roots for $\Delta \lambda^{(i)}$ when $i=2,3, \ldots$; and the same holds for $\Delta \lambda^{(1)}$ provided ${ }^{t+\Delta t} W$ is small enough and equilibrium was sufficiently well satisfied at the end of the previous load step.

2.2.4 Some general remarks. Considering the iterative schemes described in the previous sections we note that the coefficient matrix is not necessarily positive definite. If the matrix is not positive definite a critical point has been passed, but the triangular factorization can be completed, provided there is no 
multiplier growth. In practice, the triangular factorization only fails if it is attempted at, or very close to, a critical point - a situation that is hardly observed.

Once a critical point has been passed an appropriate solution for $\Delta \lambda^{(i)}$ may be a negative value, and such value is used if the coefficient matrix is not positive definite.

We also note that a number of constants have to be selected and initialized for the algorithm; namely $\alpha, \delta$ and $N_{1}$. Reasonable values for $\delta$ and $N_{1}$ are 0.15 and 6 , respectively, but effective values for $\alpha$ are problem-dependent and in the solutions reported upon in Section 4 we used $\alpha$ from 2 to 50 . If the prescribed displacement ${ }^{\Delta t} U_{k}^{*}$ is small, a large value of $\alpha$ may be appropriate. In addition more appropriate values for $\beta^{\prime}$ in eqn (32) can perhaps be selected. The proper choice of these constants does affect the performance of the solution algorithm, but more experience need be gained before more specific recommendations can be made.

\section{LINEARIZED BUCKLING ANALYSIS}

The automatic solution procedure presented in Section 2 calculates incrementally the complete solution path, including the post-collapse response. The method is quite general but, although effective, can still lead to a relatively high solution cost, because an incremental solution is performed. In some analyses for which the pre-collapse displacements are negligible it is valuable to calculate only an estimate of the buckling load of the structure, without going through a solution for the complete nonlinear response. This may for some structures be achieved economically by a linearized buckling analysis.

Let $\tau$ decide the load level at which buckling or collapse would occur in an incremental analysis, then we have

$$
\operatorname{det}\left({ }^{r} \mathbf{K}\right)=0
$$

In the linearized buckling analysis we assume that

$$
{ }^{t} \mathbf{K}={ }^{t-\Delta} \mathbf{K}+\lambda\left(\mathbf{K}-{ }^{t-\Delta} \mathbf{K}\right)
$$

where ${ }^{t-\Delta^{t}} \mathbf{R}+\lambda\left({ }^{t} \mathbf{R}-{ }^{t-\Delta} \mathbf{R}\right)$ is an approximation to the buckling load. The relation in eqn (34) can be applied at any time $\Delta t, 2 \Delta t, 3 \Delta t, \ldots$ but clearly assumes that from time $t-\Delta t$ onwards the linear strain and nonlinear strain stiffness matrices change proportionally with additional load increments. This is a severe assumption and may lead to greatly overestimating the buckling load. Hence, the results of a linearized buckling analysis must be interpreted with great care [1, 12].

Considering eqns (33) and (34) we obtain the eigenproblem

$$
{ }^{t-\Delta t} \mathbf{K} \phi=\lambda \Delta \mathbf{K} \phi
$$

where $\Delta \mathbf{K}={ }^{t-\Delta t} \mathbf{K}-{ }^{t} \mathbf{K}$ and $\Delta \mathbf{K}$ is in general indefinite. Hence, in general, the problem in eqn (35) has negative and positive eigenvalues.

Another eigenproblem for calculating the buckling load is reached by writing eqn (35) in the form

$$
\Delta \mathbf{K} \phi=\chi^{t-\Delta t} \mathbf{K} \phi
$$

where $\chi=1 / \lambda$ and - assuming that we require the smallest positive eigenvalue of eqn (35), which we call $\lambda_{1}$ - we now want to calculate the largest positive eigenvalue of eqn (36). Hence we can impose a shift $\mu=1.0$ onto the problem in eqn (36) and consider the eigenproblem

$$
{ }^{\top} \mathbf{\phi}=\gamma^{t-{ }^{\Delta}} \mathbf{K} \phi
$$

where now all eigenvalues $\gamma_{1}$ are positive and we seek the smallest positive eigenvalue $\gamma_{1}$. The critical load factor is given by

$$
\lambda_{1}=\frac{1}{1-\eta_{1}} .
$$

The effectiveness of using eqns (35) or (37) lies in that we do not calculate linear and nonlinear strain stiffness matrices separately (on the element and total assemblage levels) but instead we use the usual tangent stiffness matrices in the eigensolution. Therefore, no special programming is required to obtain the matrices for the linearized buckling analysis.

Considering eqns (35) and (37) it appears that eqn (37) is employed most effectively, because $\Delta \mathbf{K}$ does not need to be formed and all eigenvalues are positive. However, in practice we find that-depending on the structure and load level considered - the eigenvalues $\gamma_{t}$ of eqn (37) can be very closely spaced. Hence, convergence in the iterative solution using eqn (37) can be more difficult, and in that case eqn (35) is used more effectively. Also, if $\lambda_{1}$ is large, $\gamma_{1}$ is close to 1.0 and an error in $\gamma_{1}$ can yield a large error in $\lambda_{1}$.

\section{SOME SAMPLE SOLUTIONS}

The algorithms described in the previous sections have been implemented in ADINA and we present in this section some of our experiences with the methods.

For the analyses described in Sections $4.1-4.5$, in each case, the first load step displacement was imposed and then the algorithm calculated automatically the response for all subsequent load steps. as described in Section 2.

In the analyses described below we deal with somewhat simple structures whose responses, however, contain the important different features of complex analyses. We have not addressed yet the issue of obtaining most effective solutions measured on the numerical effort involved, and therefore do not report the actual computational efforts expended in the solutions. However, in no case of analysis was the computational expense much higher than when using prescribed, i.e. a priori given, load levels to solve for the response.

\subsection{Analysts of simple arch structure}

Figure 1 shows the two-bar structure considered and the solution obtained, when the structure was subjected to a compressive load (resulting in snapthrough) and a tensile load.

The performance of the automatic load stepping algorithm is interesting. We note that in the analysis of the snap-through, the algorithm switched with $\delta=0.15$ near the critical points automatically from the constant arc-length criterion to the constant external work criterion, and vice versa. On the other 

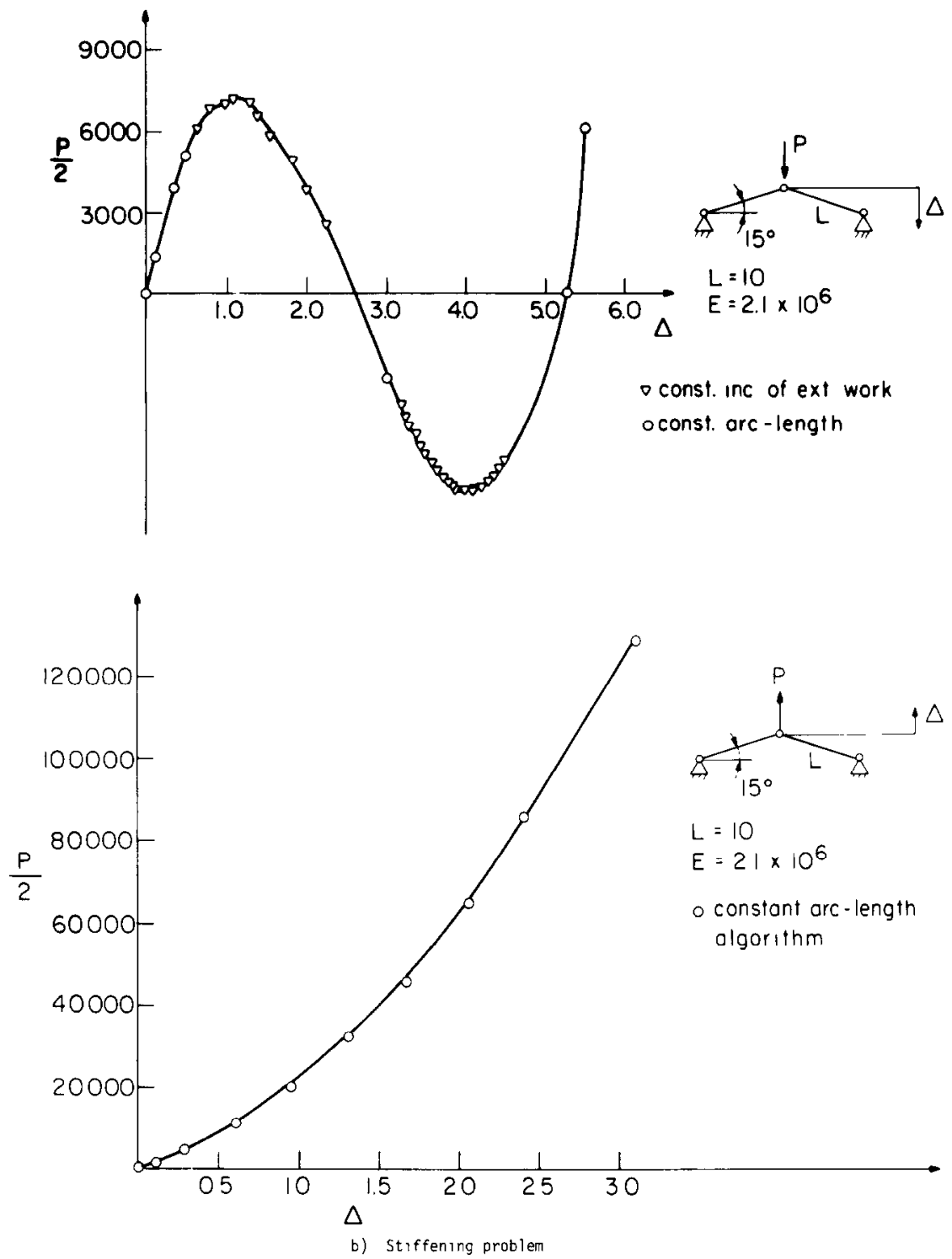

Fig. 1. Analysis of a simple arch structure using updated Lagrangian formulation[1].

hand in the analysis of the stiffening problem, the constant arc-length criterion was employed throughout and the load-steps increased during the response calculations.

\subsection{Analysis of elastic-perfectly plastic thick-walled cylinder}

Figure 2 shows the cylinder considered. This structure was analysed earlier with prescribed load levels [2]

The response calculated with the automatic load stepping algorithm is shown in Fig. 2 and is compared with the solution reported in [13].

The difficulty of calculating the collapse load with prescribed load levels lies in that very small load increments near the ultimate load must be chosenwhich for a complex structure in practice is not known-whereas the automatic load stepping algorithm computes the complete load-displacement response fully automatically.

\subsection{Large displacement analysis of cantilever}

The cantilever analyzed already in Ref. [2] provides a simple but difficult test problem.

Figure 3 shows the cantilever and the response calculated with the automatic load stepping algorithm. The total number of load steps now employed for the analysis- with a very tight convergence tolerance (ETOL $=10^{-7}$ in eqn 28) - was 50 and a total of 446 modified Newton-Raphson equilibrium iterations were performed. This compares favorably with the solution effort reported in [2].

4.4 Collapse analysis of two elastic-perfectly plastic trusses

Figures 4 and 5 show the two truss structures which were analyzed for their collapse loads. These structures were already studied earlier by Hodge and White $[14,15]$, who had difficulties with the response solutions, illustrating that the materially-nonlinearonly formulation should not be used for this kind of 

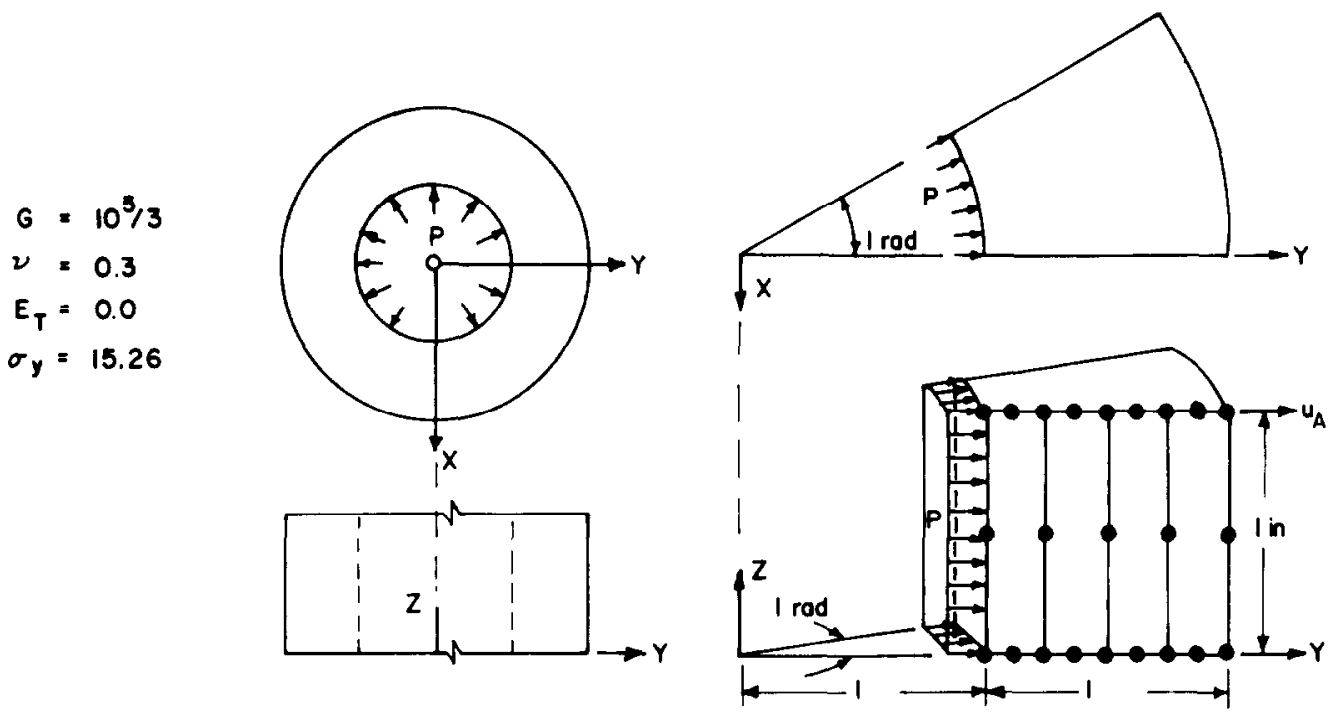

TOP AND SIDE VIEWS

ENLARGED TOP VIEW AND AXISYMMETRIC MESH

ELASTIC -PERFECTLY PLASTIC MATERIAL

PLANE STRAIN CONDITIONS

VON-MISES YIELD CONDITION

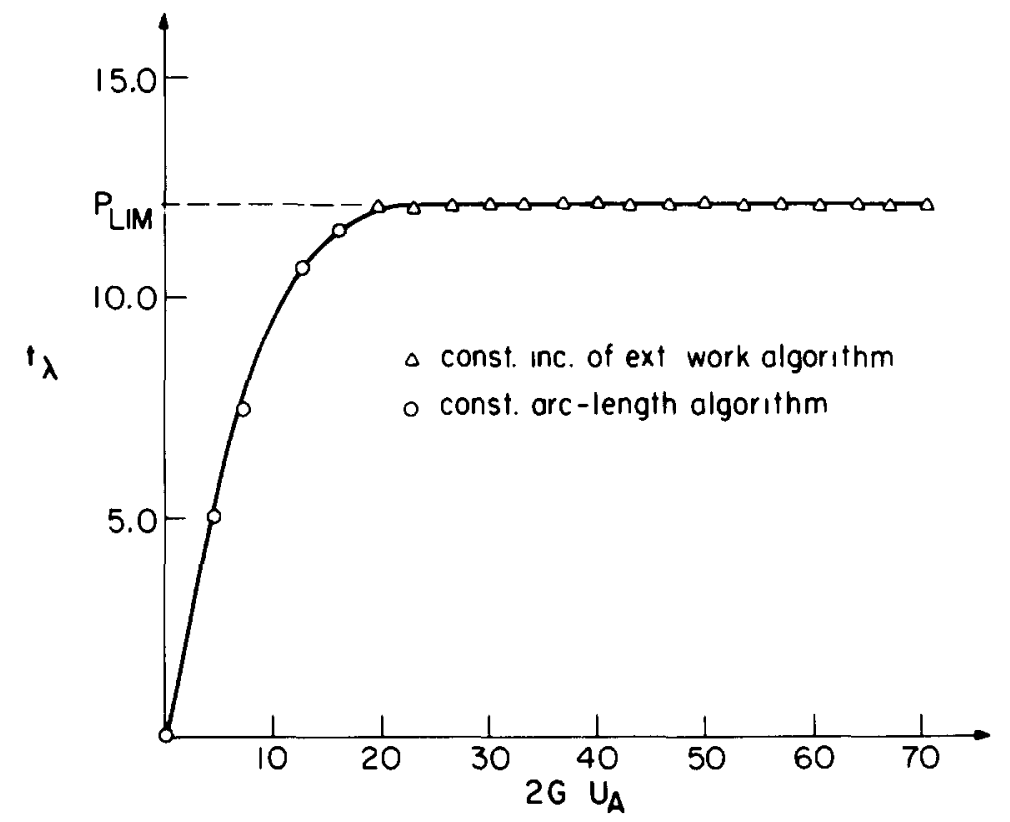

Fig. 2. Analysis of a thick-walled elastic perfectly plastic cylinder using materially-nonlinear-only or updated Lagrangian formulation.

analysis. There is no difficulty with the response solution when the updated Lagrangian formulation is used [1].

Figures 4 and 5 show the calculated response with our automatic load stepping algorithm using the updated Lagrangian formulation.

For comparison, we also calculated the response of the structures using prescribed load levels, and Table 1 summarizes the calculated values for the larger truss structure.

Note that in Figs. 4 and 5, the final slopes of the load-displacement curves are slightly positive (too small to be seen in the figures) because of the geometric stiffening effects in the models. Also, in the figures, $P_{e}$ and $P_{y}$ correspond to the analytically calculated loads at first yield and collapse, respectively. The incremental solution yields of course only a set of discrete solution points which, in the figures, have simply been connected by straight lines (points $A, B, C, D$ ).

\subsection{Structure with snap-back characteristic}

The simple elastic truss structure shown in Fig. 6 was analyzed in [9] using a displacement controlled method. Due to the snap-back characteristic, the 

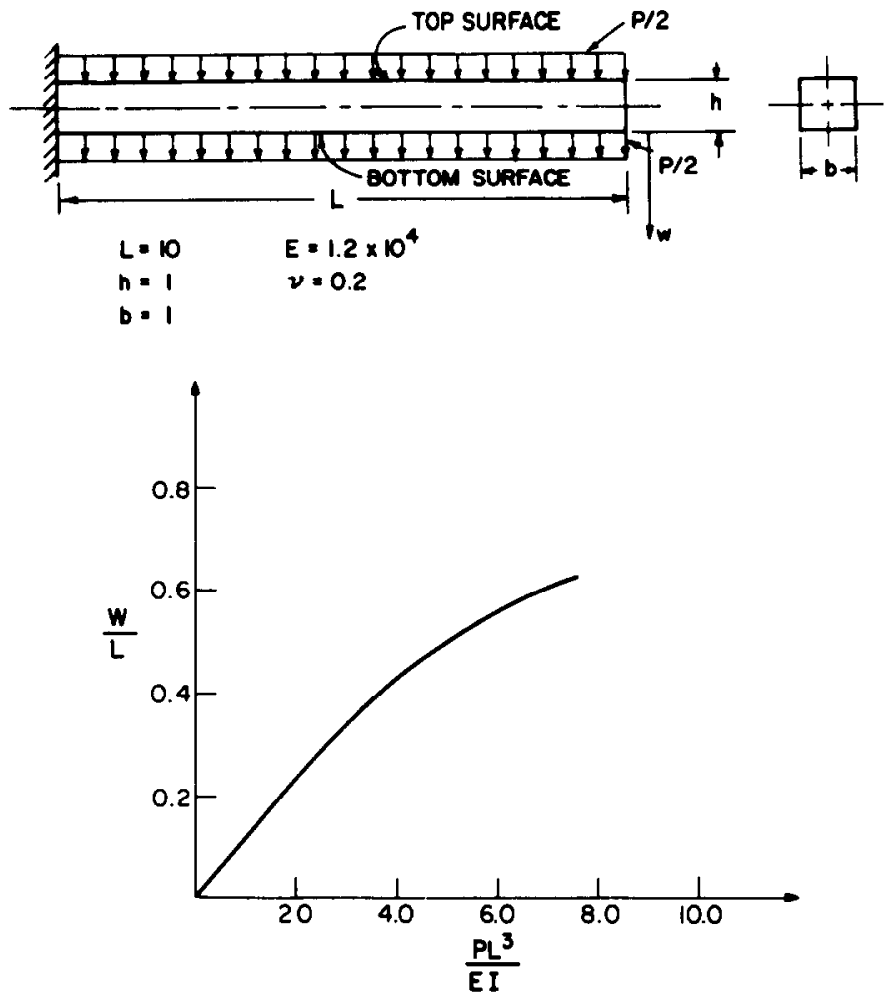

Fig. 3. Large displacement analysis of a cantilever using total Lagrangian formulation.
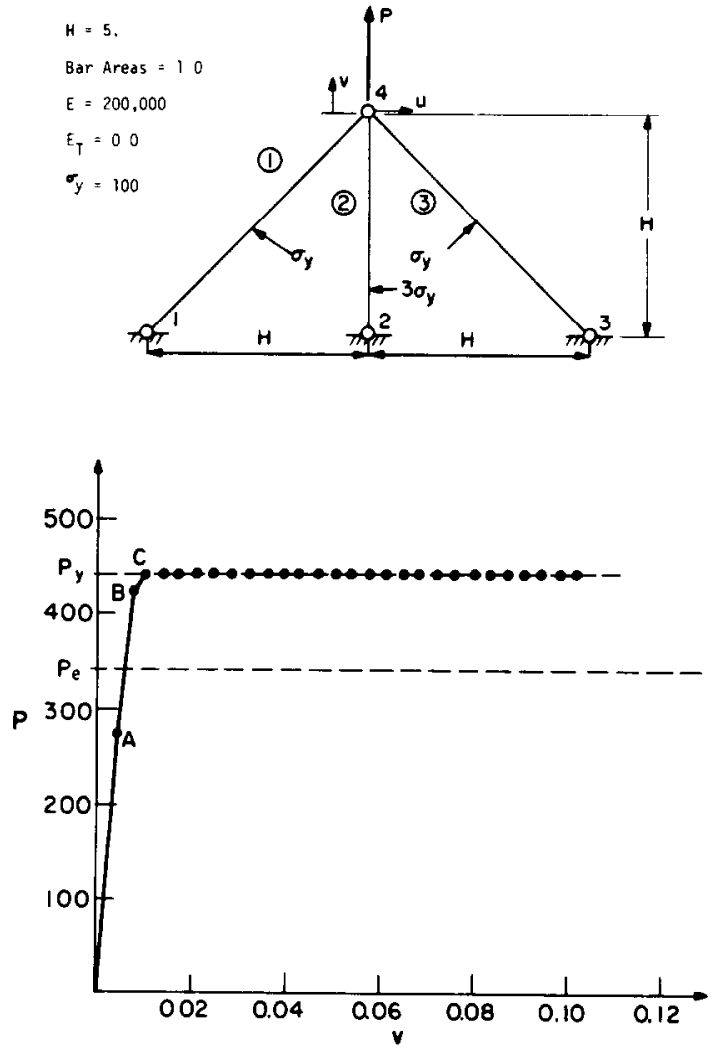

Fig. 4. Analysis of a triangular truss structure using updated Lagrangian formulation. 

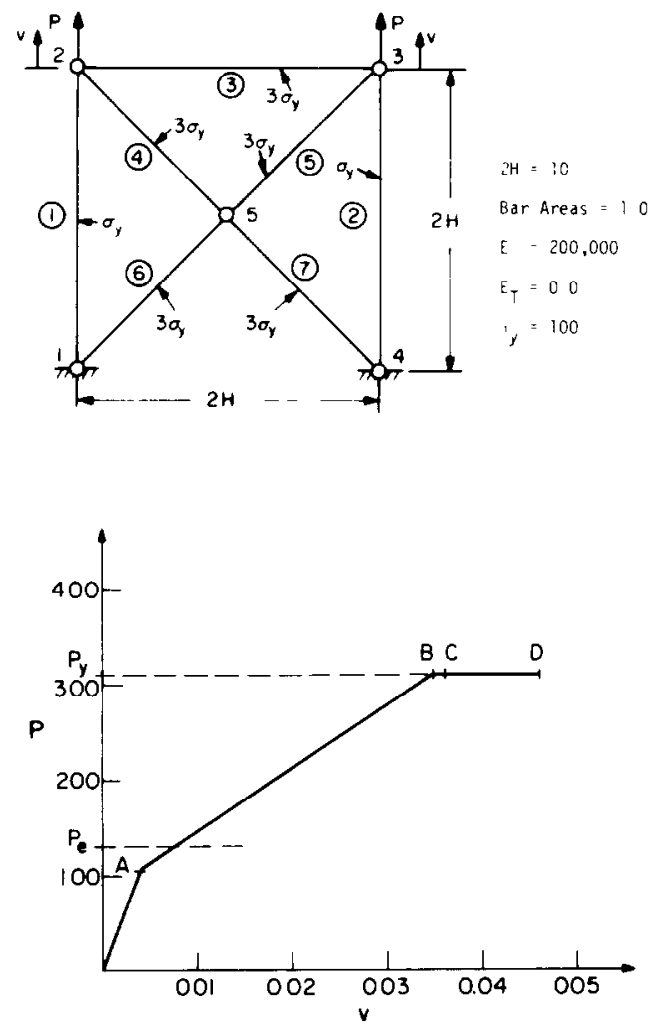

Fig. 5. Analysis of a 7-bar truss structure using updated Lagrangian formulation. displacement at node 1 could not be used as the controlling displacement.

To use the automatic algorithm, an initial displacement was imposed at node 1 and from there on, the response was automatically traced.

The solution results are given in Fig. 6 and are coincident with the solution obtained in [9].
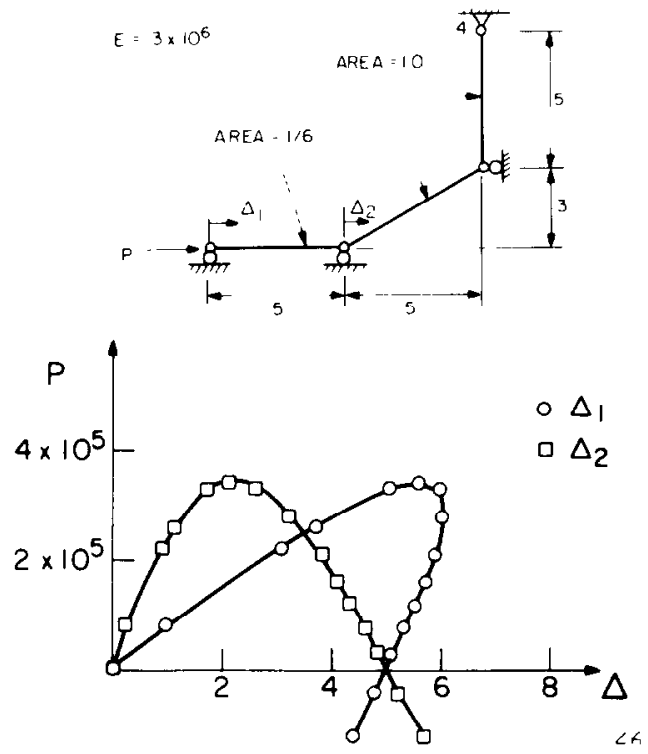

Fig. 6. Analysis of a structure with snap-back characteristic using updated Lagrangian formulation.

Table 1. Results in analysis of 7-bar truss structure

\begin{tabular}{|c|c|c|c|c|}
\hline Step & $P / P_{e}$ & $P / P_{y}$ & $\begin{array}{l}\text { Number of Iterations } \\
\text { (BFGS) }\end{array}$ & State at End of Step \\
\hline 1 & 0.38 & 0.16 & 1 & totally elastic \\
\hline 2 & 0.82 & 0.34 & 1 & " " " \\
\hline 3 & 1.00 & 0.42 & 1 & " \\
\hline 4 & 1.18 & 0.49 & 1 & bars 1,2 - plastic \\
\hline 5 & 1.36 & 0.57 & 1 & " $"$ " " n \\
\hline 6 & 1.54 & 0.64 & 1 & " " " \\
\hline 7 & 1.72 & 0.72 & 1 & " " " " \\
\hline 8 & 1.90 & 0.79 & 1 & " " " \\
\hline 9 & 2.08 & 0.87 & I & " " " " \\
\hline 10 & 2.26 & 0.94 & 1 & " \\
\hline 11 & 2.44 & 1.02 & 4 & bars $1,2,4,5,6,7$ plastic \\
\hline \multicolumn{5}{|c|}{ First analysis } \\
\hline Step & $P / P_{e}$ & $P / P_{y}$ & $\begin{array}{l}\text { Number of I terations } \\
(B F G 5)\end{array}$ & State at End of Step \\
\hline 1 & 1.00 & 0.42 & 1 & totally elastic \\
\hline 2 & 1.72 & 0.72 & 1 & bars 1,2 - plastic \\
\hline 3 & 2.44 & 1.02 & 4 & bars $1,2,4,5,6,7$ plastic \\
\hline \multicolumn{5}{|c|}{ Second analys is } \\
\hline
\end{tabular}




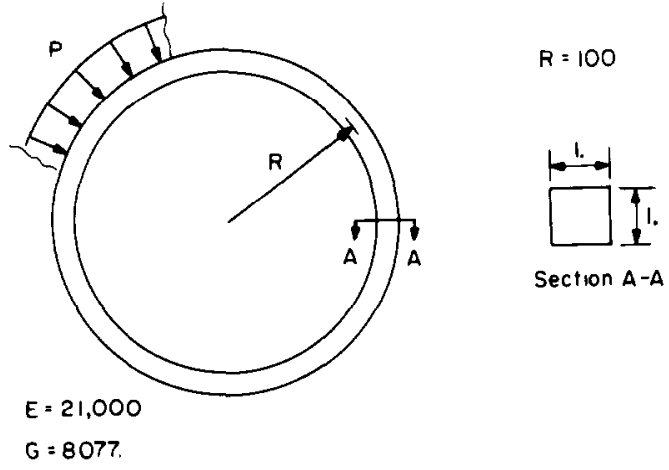

Fig. 7. Analysis of a circular ring under constant pressure.

\subsection{Linearized buckling analysis of a circular ring}

The circular ring shown in Fig. 7 was modeled using sixty 3-node isoparametric beam elements. The ring is subjected to external pressure, which is assumed not to change direction.

The analytical buckling pressure for the ring is $p_{c r}=0.7[16]$

We used eqns (35) and (37) to estimate the value of $p_{c r}$. Using 2 point Gauss integration we obtained the exact value, whereas with 3 point integration the estimated buckling load is $8.3 \%$ too high.

\section{CONCLUDING REMARKS}

The research and development reported in this paper represents some efforts towards more effective automatic incremental solution schemes for general nonlinear analysis.

The linearized buckling analysis procedure is effective because the required matrices can be calculated very economically, and the solution is then performed using either the subspace iteration method or the determinant search method[1].

The automatic load stepping algorithm has performed well in the solution of various problemssoftening, stiffening, collapse and post-buckling analyses. However, improvements in this solution procedure should still be pursued in particular with regard to the use of full Newton iteration, the BFGS method and line searches in the overall solution strategy. Also, more experience with this algorithm will be valuable in order to confirm and possibly improve the use of the various tolerances and decision making processes employed in the solution procedure.
Acknowledgements - We gratefully acknowledge the fellowship earlier provided to E. Dvorkin by the Organization of American States and the support of the ADINA users group for this research.

\section{REFERENCES}

1. K. J. Bathe, Finite Element Procedures in Engineering Analysis. Prentice-Hall, Englewood Cliffs, New Jersey (1982).

2. K. J. Bathe and A. P. Cimento. Some practical procedures for the solution of nonlinear finite element equations. J. Comput. Meth. Appl. Mech. Engng 22. 231-277 (1980).

3. E. Riks, An incremental approach to the solution of snapping and buckling problems. Int. J. Solids Structures 15, 529-551 (1979).

4. P. G. Bergan, G. Horrigmoe, B. Kräkeland and T. H. Søreide, Solution techniques for nonlinear finite element programs. Int. J. Num. Meth. Engng 12, 1677-1696 (1978).

5. E. Ramm, Strategies for tracing nonlinear responses near limit points. In Nonlinear Finite Element Analysis in Structural Mechanics (Edited by Wunderlich et al.), pp. 63-89. Springer-Verlag, New York (1981).

6. J. L. Batoz and G. Dhatt, Incremental displacement algorithms for nonlinear problems. Int. J. Num. Meth. Engng 14, 1262-1267 (1979).

7. M. A. Crisfield, A fast incremental/1terative solution procedure that handles snap-through. Comput. Structures 13, 55-62 (1981).

8. M. A. Crisfield, Variable step-lengths for nonlinear structural analysis. TRR. Lab., Rep. 1049 (1982).

9. G. Powell and J. Simons. Improved iteration strategy for nonlinear structures. Int. J. Num. Meth. Engng 17, 1455-1467 (1981)

10. H. Rothert, T. Dickel and D. Renner, Snap-through buckling of reticulated space trusses. $A S C E, J$. Struct. Div. 107, 129-143 (1981).

11. J. Padovan and S. Tovichakchaikul, On the solution of elastic-plastic static and dynamic post-buckling collapse of general structure. Comput. Structures 16, 199-205 (1983).

12. B. Brendel and E. Ramm, Nichtlineare Stabilitätsuntersuchungen mit der Methode der Finiten Elemente. Ingenieur-Archiv 51, 337-362 (1982)

13. W. Prager and P. G. Hodge, Theory of Perfectly Plastic Solids. Dover Publications, New York (1968)

14. D. L. White and P. G. Hodge, Computation of nonunique solutions of elastic-plastic trusses. Comput. Structures 12, 769-776 (1980).

15. P. G. Hodge and D. L. White, Non-uniqueness in contained plastic deformation. J. Appl. Mech. 47, 273-277 (1980).

16. G. A. Wempner and N. E. Kest1, On the buckling of circular arches and rings. Proc. 4th U.S. Nat. Cong. Appl. Mech. 2, A.S.M.E. (1962). 